\title{
Descripción osteológica del puma andino (Puma concolor): II. Esqueleto axial
}

\author{
Osteological description of the Andean puma (Puma concolor): II. Axial skeleton
}

\author{
Celso Zapata ${ }^{1,3}$, Joel I. Pacheco ${ }^{2,4}$
}

\section{Resumen}

Se estudiaron dos esqueletos axiales de puma (Puma concolor), uno macho y otro hembra, que fueran decomisados en la provincia de Canchis (Perú) y cedidos al Centro de Investigación IVITA en virtud del Acta de Custodia Temporal N. ${ }^{\circ}$ 01-2015-SERFORATFFS-Cusco, y con autorización de investigación $\mathrm{N}^{\circ}$ 158-2015-SERFOR-DGGSPFFS. Se utilizó el método estándar para la disección del esqueleto y los términos utilizados están de acuerdo con la Nómina Anatómica Veterinaria. Las mediciones se realizaron con un calibrador vernier. A nivel del cráneo y mandíbula destacan las eminencias y depresiones relacionadas con el origen e inserción de músculos, tales como la cresta nucal, cresta sagital, la fosa masetérica y la fosa del parietal. La columna vertebral en conjunto asemeja a lo descrito en los gatos, a excepción de ciertas particularidades que son más prominentes, como la apófisis accesoria y la apófisis mamilar. A nivel de las vértebras torácicas, la vértebra anticlinal es la T11, teniendo esta una apófisis espinosa de menor longitud. Las costillas y el esternón siguen los patrones descritos para los demás felinos.

Palabras clave: puma; esqueleto axial; cráneo; vertebras; costillas

\section{AbSTRaCt}

Two axial skeletons of puma (Puma concolor), one male and one female, that were confiscated in the province of Canchis (Peru) and ceded to the IVITA Research Centre under the Temporary Custody Act No. 01-2015-SERFOR-ATFFS-Cusco were used through the research authorization $\mathrm{N}^{\circ} 158-2015$-SERFOR-DGGSPFFS. The standard method for

\footnotetext{
${ }^{1}$ Escuela de Medicina Veterinaria, Universidad Nacional San Antonio Abad del Cusco, Perú

${ }^{2}$ Grupo de Investigación en Fauna Silvestre, Estación Maranganí, Centro de Investigación IVITA, Universidad Nacional Mayor de San Marcos, Cusco, Perú

${ }^{3}$ E-mail: czapatacoacalla@gmail.com

${ }^{4}$ E-mail:jpachecoc@unmsm.edu.pe
}

Recibido: 22 de mayo de 2018

Aceptado para publicación: 29 de noviembre de 2018 


\begin{abstract}
the dissection of the skeleton was applied and the terms used are in accordance with the Veterinary Anatomical Terminology. The measurements were made with a calliper. At the level of the skull and jaw, the eminences and depressions related to the origin and insertion of muscles stand out, such as the nuchal crest, sagittal crest, the masseteric fossa and the parietal fossa. The vertebral column resembles that described in cats, except for certain particularities that are more prominent, such as the accessory process and the mamillary process. At the level of the thoracic vertebrae, the anticlinal vertebra is T11, this having a shorter spinous process. The ribs and the sternum follow the patterns described for other felines.
\end{abstract}

Key words: puma; axial skeleton; skull; vertebrae; ribs

\section{INTRODUCCIÓN}

Se estima que el Perú alberga 508 especies de mamíferos, incluyendo 35 especies de carnívoros (Pacheco et al., 2009). En el orden Carnivora (Mammalia) se tiene al Puma concolor (Cossíos et al., 2012), conocido tambien con el nombre de puma, león, lluichupuma, kirajari malsonsori (Pacheco et al., 1995), siendo categorizado como Casi Amenazados (NT) en la «Lista de clasificación y categorización de las especies amenazadas de fauna silvestre legalmente protegidas» aprobado por el DS N. ${ }^{\circ} 004-2014-M I N A G R I$ (Ministerio de Agricultura y Riego, 2014).

Este puma se encuentra en una amplia gama de hábitats, teniendo problemas por la pérdida constante de sus hábitats, así como por la caza furtiva de su presa silvestre; además, son perseguidos en represalia a la depredación del ganado y al temor que representa como amenaza para la vida humana (Nielsen et al., 2015). Por otro lado, resalta su importancia como regulador de las poblaciones presa (Hernández-Guzmán et al., 2011).

Existe grandes vacíos de información relevante para la conservación de las especies del orden Carnívora (Cossíos et al., 2012), donde las revisiones taxonómicas modernas empleando descripciones morfológicas, cariotípicas y moleculares son una ne- cesidad de primer orden (Pacheco et al., 2009). Es así que el presente estudio realiza una descripción anatómica del esqueleto axial del Puma concolor con el fin de contribuir al mejor conocimiento de la especie.

\section{Materiales y Métodos}

Se utilizaron esqueletos axiales de dos pumas andinos adultos. Un macho de $52 \mathrm{~kg}$ y una hembra de $45 \mathrm{~kg}$ de peso, los que fueran decomisados en las provincias de Canchis y Canas, respectivamente, departamento de Cusco (Perú), y remitidos al Laboratorio de Fauna Silvestre de la Estación Experimental del Centro de Investigación IVITA de la Universidad Nacional Mayor de San Marcos, ubicada en Maranganí, Cusco. El decomiso y envío al laboratorio se hizo por el Servicio Nacional Forestal y de Fauna Silvestre (SERFOR), mediante Acta de Custodia Temporal N. ${ }^{\circ}$ 01-2015-SERFOR-ATFFS-Cusco. El presente trabajo se encuentra amparado mediante la autorización de investigación $\mathrm{N}$. $^{\circ}$ 158-2015-SERFOR-DGGSPFFS.

Se utilizó la técnica descrita por Cañete et al. (2014) para la disección y obtención del esqueleto. Para la descripción del esqueleto axial se utilizaron las pautas descritas para carnívoros por Getty et al. (1990) y Dyce et al. (2007). Para las medidas de las piezas óseas se utilizó lo descrito por Von Den 
Cuadro 1. Medidas ( $\mathrm{cm}$ ) de la cabeza del puma andino (Puma concolor). Un macho y una hembra

\begin{tabular}{lcc}
\hline & \multicolumn{2}{c}{ Medidas (cm) } \\
\cline { 2 - 3 } & Macho & Hembra \\
\hline Ancho a nivel del zigomático & 13.2 & 12 \\
Máximo ancho del neurocráneo & 7.3 & 6.8 \\
Longitud del cóndilo basal & 17.1 & 16.2 \\
Máximo ancho palatal & 7.8 & 7.6 \\
Longitud de la fila premolar & 4.3 & 4.1 \\
Máxima longitud interior de la órbita & 4.1 & 3.8 \\
Altura de la rama vertical & 6.3 & 5.7 \\
Longitud total & 12.1 & 11 \\
Longitud de la fila premolar, molar & 4.4 & 4.3 \\
\hline
\end{tabular}

Driesch (1976). Los términos utilizados están de acuerdo con la Nómina Anatómica Veterinaria (Schaller, 1996).

\section{Resultados}

\section{Cabeza}

Los huesos de la cabeza se describen a continuación. Las medidas se encuentran en el Cuadro 1.

- Occipital: la cresta nucal es prominente, de allí hacia rostral se dirige una cresta sagital pronunciada. Las apófisis yugulares son cortas.

- Parietal: Forma la mayor parte del techo de la cavidad craneana. La superficie externa es convexa. y presenta la cresta sagital que se bifurca, una a cada hueso frontal

- Frontal: hueso corto y ligeramente convexo. Presenta apófisis cigomáticas cortas. No se encuentra el agujero supraorbitario. La superficie orbital es amplia.
- Temporal: la apófisis cigomática es amplia y curva constituyendo el extremo transversal más amplio. Presenta una superficie articular para los cóndilos de la mandíbula que es amplia y transversal. La bulla timpánica es grande, redonda y lisa, presentando una pequeña apófisis mastoidea.

- Maxilar: es corto y alto. Presenta el canal infraorbitario y un gran alveolo para alojar al diente canino.

- Incisivos: cuerpo aplanado dorsoventralmente con tres alveolos dentarios para los incisivos, la fisura palatina es grande y casi circular.

- Palatinos: la lámina horizontal es amplia conformando casi un tercio del paladar duro. Presentan un foramen palatino mayor. La lámina vertical presenta una apófisis pterigoidea pequeña.

- Pterigoideos: presentan una apófisis pterigoidea delgada y ganchosa.

- Lacrimal: es muy pequeño y presenta la entrada del canal lacrimal.

- Cigomático: es largo y curvo presentan cresta facial y una apófisis frontal del hueso cigomático pequeño. 
Cuadro 2. Medidas de la máxima anchura sobre las alas (GB), máxima longitud (GL), altura (H), máxima longitud alrededor del cuerpo (LCDe), máxima longitud de los procesos articulares craneales a los procesos articulares caudales (GLPa) en (cm) de las vértebras cervicales del puma andino (Puma concolor)

\begin{tabular}{lcccccccc}
\hline & & \multicolumn{8}{c}{ Vértebras cervicales } \\
\cline { 3 - 9 } & & Atlas & Axis & C3 & C4 & C5 & C6 & C7 \\
\hline Macho & GB & -- & -- & -- & -- & -- & -- & -- \\
& GL & -- & -- & -- & -- & -- & -- & -- \\
& H & -- & -- & 3.8 & 3.7 & 3.8 & 3.3 & 6.4 \\
& LCDe & -- & 6.2 & -- & -- & -- & -- & -- \\
& GLPa & -- & -- & 4.0 & 4.0 & 3.4 & 5.1 & 3.3 \\
\hline \multirow{7}{*}{ Hembra } & GB & 7.4 & -- & -- & -- & -- & -- & -- \\
& GL & 4.3 & -- & -- & -- & -- & -- & -- \\
& H & 2.6 & -- & 2.7 & 3.4 & 3.7 & 4.2 & 6.5 \\
& LCDe & -- & 5.5 & -- & -- & -- & -- & -- \\
& GLPa & -- & -- & 3.7 & 3.2 & 2.9 & 2.8 & 3.1 \\
\hline
\end{tabular}

- Mandibula: presentan sínfisis mandibular. La fosa masetérica profunda, apófisis angular manifiesta. La apófisis condilar amplia transversalmente y la apófisis coronoidea corta y ancha.

- Dientes: I 3/3 C 1/1 PM 3/2 M 0/1

\section{Vértebras Cervicales}

Las vértebras cervicales se describen a continuación. Las medidas se observan en el Cuadro 2.

- Atlas. El arco ventral estrecho presenta una pequeña tuberosidad ventral. El arco dorsal es ancho y presenta rugosidades en su superficie dorsal. La relación entre ambos es de 1:2. Las alas son anchas, horizontales y el borde libre es convexo. La escotadura alar está bien definida. La superficie articular para los cóndilos del occipital están bien definidos, separados y cóncavos. La superficie articular para el axis es ligeramente plano y presenta foramen transverso y lateral.
- Axis. El cuerpo es aplanado dorsoventralmente. Presenta cresta ventral poco prominente. La apófisis odontoide es cilíndrica, larga, inclinada ligeramente dorsalmente y abarca la longitud del arco ventral del atlas. Las apófisis transversas puntiagudas dirigidas caudal y lateralmente, atravesadas por el agujero transverso. La apófisis espinosa ligeramente alta y larga, y cranealmente se proyecta hasta el atlas, cubriendo el espacio interarcual. Caudalmente presenta un tubérculo que se une con las apófisis articulares caudales. Su borde libre es ligeramente grueso y rugoso.

- 3ra, 4ta, 5ta vértebra cervical. Vértebras cortas con relación a las primeras. El cuerpo presenta una cresta ventral, las apófisis transversas presentan dos porciones, una craneal y una caudal, atravesadas por el foramen transverso. Apófisis articulares cortas con superficie articular plana. En las apófisis articulares caudales presentan una eminencia que se dirige caudalmente, perdiéndose en la quinta vér- 
Cuadro 3. Altura $(\mathrm{cm})$ de las vértebras torácicas del puma andino (Puma concolor)

\begin{tabular}{lccccccccccccc}
\hline & T1 & T2 & T3 & T4 & T5 & T6 & T7 & T8 & T9 & T10 & T11 & T12 & T13 \\
\hline Macho & 7.8 & 8.4 & 8.3 & 8.1 & 7.8 & 7.4 & 6.7 & 6.2 & 5.8 & 5.1 & 3.4 & 4.9 & 5.0 \\
Hembra & 7.2 & 7.7 & 7.6 & 6.9 & 7.1 & 6.4 & 6.6 & 5.5 & 4.9 & 3.8 & 3.9 & 4.4 & 4.4 \\
\hline
\end{tabular}

Cuadro 4. Altura $(\mathrm{H})$ y ancho máximo a través de los procesos transversos $(\mathrm{BPtr})(\mathrm{cm})$ de las vértebras lumbares del puma andino (Puma concolor).

\begin{tabular}{lcccccccc}
\hline & & L1 & L2 & L3 & L4 & L5 & L6 & L7 \\
\hline Macho & H & 5.1 & 5.5 & 5.9 & 6.4 & 6.4 & 6.1 & 5.8 \\
& BPtr & 4.9 & 5.5 & 6.2 & 6.5 & 7.4 & 7.5 & 7.4 \\
Hembra & H & 4.8 & 5.0 & 5.2 & 5.5 & 5.4 & 5.4 & 5.2 \\
& BPtr & 4.9 & 5.8 & 5.7 & 5.6 & 6.4 & 7.4 & 7.1 \\
\hline
\end{tabular}

tebra cervical. Las apófisis espinosas aumentan de longitud de la tercera a la quinta vértebra, dirigiéndose hacia craneal.

- Gta vértebra cervical. Apófisis espinosa dirigida hacia dorsal y craneal. Las apófisis transversas presentan tres porciones: dos dirigidas hacia ventral y lateral, y otra dirigida hacia lateral. Las apófisis articulares caudales poco manifiestas y las craneales bien prominentes.

- $7 m a$ vértebra cervical. Es la más corta. Su apófisis espinosa es la más larga, dirigida hacia dorsal y craneal. Presenta una sola porción en las apófisis transversas. A nivel del cuerpo en el extremo caudal no existe una verdadera superficie articular para la cabeza de la primera costilla.

\section{Vértebras Torácicas}

Son en número de 13. Las medidas de la altura de las vértebras se encuentran en el Cuadro 3. Las 10 primeras vértebras torácicas presentan un cuerpo ligeramente aplanado dorsoventralmente, con presencia de una cresta ventral. Las apófisis espinosas se encuentran dirigidas dorsal y caudalmente, el extremo libre es grueso y rugoso, y decrecen en su longitud hacia caudal. Las superficies articulares craneales son amplias y ocupan cerca de la mitad del arco dorsal, y las superficies articulares caudales se ubican en la base de la apófisis espinosa. La primera vértebra presenta superficies articulares craneales bien manifiestas con superficies articulares mirando hacia dorsal. De la primera a la novena vértebra presentan una pequeña apófisis mamilar. La décima y onceava vértebra presentan una apófisis mamilar bien desarrollada, dividiéndose en dos porciones: una craneal y otra caudal. La cabeza de las costillas se articula con los dos cuerpos de las vértebras.

La vértebra anticlinal es la vértebra 11. No presenta apófisis espinosa manifiesta. Las apófisis articulares caudales se dirigen hacia caudal y dorsal. Las superficies articulares miran hacia lateral, presentan apófisis mamilar bien pronunciada y una apófisis accesoria manifiesta. A nivel del cuerpo presenta su- 
Cuadro 5. Anchura máxima sobre las alas (GB) y longitud máxima (GL) (cm) del sacro del puma andino (Puma concolor)

\begin{tabular}{lcc}
\hline Sexo & $\begin{array}{c}\text { Anchura } \\
\text { máxima sobre } \\
\text { las alas }\end{array}$ & $\begin{array}{c}\text { Longitud } \\
\text { máxima }\end{array}$ \\
\hline Macho & 4.8 & 6.4 \\
Hembra & 4.6 & 5.9 \\
\hline
\end{tabular}

perficie articular para la cabeza de la costilla, pero solo en la porción craneal.

Las vértebras 12 y 13 presentan apófisis espinosas que se dirigen hacia dorsal y craneal y son cortas. La apófisis mamilar y accesoria es bien manifiesta. Solo presentan una superficie articular para la cabeza de las costillas en la parte craneal.

\section{Vértebras Lumbares}

Presentan siete vértebras lumbares. El cuerpo es más largo, se aplana dorsoventralmente a medida que se acerca al sacro. Las apófisis espinosas son de la misma longitud y se dirigen hacia dorsal y craneal. Las apófisis accesorias se observan hasta la sexta vértebra lumbar. Las apófisis transversas presentan curvas largas y dirigidas hacia lateral y ventral. Las apófisis articulares son características de este tipo de vértebra, donde en el cuerpo de la vértebra 4,5 y 6 presentan una manifiesta cresta ventral. Las medidas de altura y ancho máximo de las vértebras se muestran en el Cuadro 4.

\section{Vértebras Sacras}

Formado por tres vértebras con dos forámenes dorsales y dos forámenes ventrales. Las apófisis espinosas decrecen hacia caudal. Las alas son cortas y la superficie articular vertical y miran hacia lateral.
Las medidas de ancho máximo y longitud máximo de las vértebras se presentan en el Cuadro 5.

\section{Vértebras Caudales}

Son en número de 16. Las cuatro primeras vértebras se articulan por medio de las apófisis articulares craneales y caudales y una apófisis transversa bien manifiesta. Las vértebras 5 a la 16 se articulan por medio de su cuerpo. No poseen canal vertebral ni apófisis espinosas y transversas manifiestas. A nivel del cuerpo presentan un par de tubérculos donde se ubican las apófisis hemales.

\section{Costillas}

Presentan 13 pares de costillas. En términos generales, los cinco primeros pares presentan cuerpos aplanados y en los demás, los cuerpos son ligeramente cilíndricos. Los tubérculos se articulan con las apófisis transversas en las once primeras costillas, mientras que las dos últimas no se articulan con estas apófisis.

\section{Esternón}

Formado por ocho esternebras comprimidas lateralmente, con una apófisis del manubrio bien manifiesto y el cartílago xifoides.

\section{Huesos auditivos e hioides}

Por las condiciones que fueron entregados los especímenes no fue posible recuperar estos huesos.

\section{Discusión}

Las características descritas de los huesos del cráneo, en especial aquellas relacionadas con la inserción de músculos, son similares a los descritos por Segura y Flores (2009), indicando que las modificaciones observadas tienden al fortalecimiento de la musculatura cervical. Entre estos, destacan los 
cambios osteológicos como el presentar un proceso supraorbital, cresta sagital y cresta nucal altamente desarrollados.

Los hallazgos a nivel de la mandíbula, en especial de la fosa masetérica, llegan a tener relación con los hallazgos ontogénicos encontrados por Giannini et al. (2010), donde la longitud de la mandíbula y las dos dimensiones del proceso coronoide son fuertemente alométricas, apuntando a un alargamiento del cuerpo mandibular y una ampliación de la rama mandibular. Esto sugiere un fuerte aumento en la influencia de la musculatura masticatoria en el adulto a través del área aumentada de inserción muscular, específicamente para los músculos temporales y maseteros. La alometría negativa de la altura de la mandíbula implica que se logra una alta fuerza mecánica al principio de la ontogenia. Tambien es muy notoria la presencia de sínfisis mandibular, que Scapino (1981) la cataloga como clase III, siendo estas sínfisis bastante rígidas. El maxilar es corto y alto, similar al gato (Dyce et al., 2007).

Las características de las vértebras cervicales, torácicas, lumbares y sacras siguen los patrones descritas por Dyce et al. (2007) y Getty et al. (1990), con algunas diferencias con respecto a la longitud de las apófisis mamilares y accesorias que son más grandes y sobresalientes. Las apófisis accesorias se inician desde la T10 y se extiende hasta la L7, mientras que las apófisis mamilares están presentes en todas las vértebras torácicas y lumbares. Dichos rasgos pueden estar relacionados con lo descrito por Olivares y Rojas (2013), que indican que los huesos de la columna vertebral ofrecen un lugar para la fijación de la musculatura regional e impide el acortamiento del cuerpo y soporta gran parte del peso. Así mismo, Dyce et al. (2007) indican que la vértebra anticlinal en carnívoros es la undécima, dato que cooincide con lo hallado en el puma, exceptuando que su apófisis espinosa es de menor altura (Cuadro 3 ).

\section{Conclusiones}

- La cabeza del puma andino presenta características importantes en lo referente a particularidades relacionadas a origen e inserción de músculos.

- Las vértebras en general se asemejan a lo descrito en gatos.

- A nivel de las vértebras torácicas, la anticlinal es la T11.

\section{Literatura Citada}

1. Cañete G, Sánchez J, Noda L. 2014. Ensamblaje artesanal de un esqueleto canino mediante variantes de la osteotecnia. REDVET 15(9): 1-15. [Internet]. Disponible en: http:// www.redalyc.org/articulo.oa?id=63632727005

2. Cossíos E, Alcázar P, Fajardo U, Chávez K, Alfaro-Shigueto J, Cárdenas-Alayza S, et al. 2012. El orden Carnivora (Mammalia) en el Perú: estado del conocimiento y prioridades de investigación para su conservación. Rev Peru Biol 19: 17-26. doi: 10.15381/ rpb.v19i1.783

3. Dyce K, Sack W, Wensing C. 2007. Anatomía veterinaria. $3^{\circ}$ ed. México: Ed Manual Moderno. $920 \mathrm{p}$.

4. Getty R, Sisson S, Grosman J. 1990. Anatomía de los animales domésticos. $5^{\circ}$ ed. Barcelona: Salvat. 2302 p.

5. Giannini NP, Segura V, Giannini MI, Flores D. 2010. A quantitative approach to the cranial ontogeny of the puma. Mamm Biol 75: 547-554. doi: 10.1016/ j.mambio.2009.08.001

6. Hernández-Guzmán A, Payán E, Monroy-Vilchis O. 2011. Hábitos alimentarios del puma concolor (Carnivora: Felidae) en el Parque Nacional Natural Puracé, Colombia. Rev Biol Trop 59: 1285-1294. 
7. Ministerio de Agricultura y Riego. 2014. Normas Legales Diario el Peruano 520497. [Internet]. Disponible en: https://www.serfor.gob.pe/wp-content/ uploads/2016/09/DS-N004-Especiesamenazadas-de-fauna-silvestre.pdf

8. Nielsen C, Thompson D, Kelly M, Lopez-Gonzalez C. 2015. Puma concolor. The IUCN Red List of Threatened Species 2015: e.T18868A97216466. [Internet]. Disponible en: http:/ /dx.doi.org/10.2305/IUCN.UK.20154.RLTS.T18868A50663436.en

9. Olivares R, Rojas M. 2013. Esqueleto axial y apendicular de vertebrado. Int J Morphol 31: 378-387. doi: 10.4067/ S0717-95022013000200003

10. Pacheco V, de Macedo $H$, Vivar $E$, Ascorra C, Arana-Cardó R, Solari S. 1995. Lista anotada de los mamíferos peruanos. Occasional Papers in Conservation Biology $N^{o}$ 02. p 1-35. EEUU. [Internet]. Disponible en: https://
www.academia.edu/1323769/Lista_anotada_-de_los_mam\%-C3\%ADferos_peruanos

11. Pacheco V, Cadenillas $R$, Salas $E$, Tello C, Zeballos H. 2009. Diversidad y endemismo de los mamíferos del Perú. Rev. Peru Biol 16: 5- 032. doi: 10.15381/ rpb.v16il.111

12. Scapino R. 1981. Morphological investigation into functions of the jaw symphysis in carnivorans. J Morphol 167: 339-375. doi: 10.1002/jmor. 1051670308

13. Schaller R. 1996. Nomenclatura anatómica veterinaria ilustrada. Zaragoza, España: Ed Acribia. $622 \mathrm{p}$

14. Segura V, Flores D. 2009. Aproximación cualitativa y aspectos funcionales en la ontogenia craneana del Puma concolor (Feliade). Mastozool Neotrop 16: 169-182.

15. Von Den Driesch A. 1976. A guide to the measurement of animal bones from archaeological sites. Massachusetts, USA: Harvard University. 136 p 